\title{
Efficacy of nutritional interventions supplementing nuts on cardiovascular risk factors among adults individuals: A systematic review and meta-analysis of intervention studies
}

\author{
F Liang, J Young and J Lara \\ Department of Applied Sciences, Faculty of Health and Life Sciences, University of Northumbria at Newcastle, \\ NE18ST, UK.
}

Current evidence suggests that the prevalence of healthy ageing is relatively ${ }^{(1)}$. Ageing is characterised by the degree to which body functions decline, including declines in cognition, physical capability, physiological and metabolic functions, and psychosocial wellbeing $^{(2)}$. Among the physiological and metabolic changes occurring with ageing, the cardiovascular ageing is a key determinant of health. Since cardiovascular diseases (CVDs) remain the leading cause of mortality in Europe ${ }^{(3)}$, improving CV health is a priority for research. Epidemiological evidence suggests an association between consumption of nuts and lower risk for CVDs. However, how factors such as ethnicity plays a role in this association are not well established. Evidence shows that CVD morbidity and mortality in mainland China are increasing persistently ${ }^{(4)}$ but seem to be lower among Chinese immigration in the UK ${ }^{(5,6)}$. Here we present a systematic review and meta-analysis of the evidence on the effect of supplementing nuts on CV risk factors in different ethnic groups - Asian and non-Asian population.

Databases including Medline, Web of Science and Scopus were searched from inception to August 2017. Inclusion criteria: RCTs reporting effects of nuts on $\mathrm{CV}$ risk factors among adults. The outcomes of interest included blood lipids, endothelial function, blood pressure and inflammatory factors. Random-effects models were used to determine the pooled effect sizes. The protocol for this systematic review was registered in PROSPERO (CRD42018089055). From a total of 383 publications identified, 82 studies were included in the meta-analysis. Overall, nuts intervention (with daily consumption ranging around between $4 \mathrm{~g}$ and $128 \mathrm{~g}$ ) resulted in a significant improvement in total cholesterol (mean difference: $-7.54 ; 95 \% \mathrm{CI}:-10.2$ to $-4.89 ; \mathrm{p}<0.001 ; \mathrm{I} 2=59 \% ; \mathrm{n}=66$ trials), HDL-cholesterol $(0.89 ; 95 \%$ CI: 0.04 to $1.75 ; \mathrm{P}=0.04 ; \mathrm{I} 2=53 \% ; \mathrm{n}=67$ trials), LDL-cholesterol $(-7.21 ; 95 \% \mathrm{CI}:-9 \cdot 38$ to -5.04 ; $\mathrm{P}<0.001 ; \mathrm{I} 2=68 \% ; \mathrm{n}=68$ trials $)$, triglycerides $(-8 \cdot 83 ; 95 \% \mathrm{CI}:-13.12$ to $-4.53 ; \mathrm{P}<0.001 ; \mathrm{I} 2=64 \% ; \mathrm{n}=65$ trials $), \mathrm{VLDL}-$ cholesterol $(-2.25 ; 95 \% \mathrm{CI}:-3.74$ to $-0.77 ; \mathrm{P}=0.003 ; \mathrm{I} 2=0 \% ; \mathrm{n}=10$ trials $)$, Apolipoprotein $\mathrm{B}(-4.47 ; 95 \% \mathrm{CI}:-7.01$ to $-1.94 ; \mathrm{P}=0.0005 ;$ $\mathrm{I} 2=64 \%, \mathrm{n}=23$ trials) and flow-mediated dilatation $(0.74 ; 95 \% \mathrm{CI}: 0.09$ to $1.39 ; \mathrm{P}=0.03 ; \mathrm{I} 2=5 \%, \mathrm{n}=10$ trials $)$ as compared to controls, respectively. Subgroup analysis based on ethnicity indicated that nuts consumption tended to exert a better improvement on oxidized-LDL: -6.65 (95\% CI:-29.44 to 16.14), Systolic blood pressure: -0.67 (95\% CI:-4.57 to 3.23), Apo B: -9.54 (95\% CI: -32.8 to 13.71$)$, C-reactive protein: -1.29 (95\% CI: -2.98 to 0.4$)$, TNF- $\alpha$ : -0.06 (95\% CI: -0.13 to 0.01$)$ and sVCAM-1: -48.61 (95\% CI: -97.96 to 0.74$)$ among the Asian group compared with the non-Asian population although such differences were not statistically significant.

These results provide evidence on the beneficial effects of nuts on blood lipids, lipids particles and endothelial function. In addition, the Asian group potentially appears to benefit more from nut consumption than non-Asian group. These results provide valuable knowledge for designing future human trials. However, interventions with specific nut types and ethnicity division of long duration will be needed in the future research. Such studies should also specifically focus on identifying the different efficacy of specific type of nut on both ethnicity groups.

1. Hank KJ \& Gerontol B (2011) Psychol Sci Soc Sci 66(2), 230-6.

2. Lara J et al. (2013) Maturitas 76(2), 189-99.

3. Townsend N et al. (2016) Eur Heart J 37(42), 3232-45

4. Li H, Ge J (2015) IJC Heart \& Vasculature 6, 25-31.

5. Bansal N et al. (2013) BMJ Open 3(9), e003415

6. Bhopal RS et al. (2012) Eur J Prev Cardiol 19(6), 1250-7. 